Удк 542.63; 676.054.17

\title{
ОПРЕДЕЛЕНИЕ ЭФФЕКТИВНОСТИ И ИНТЕНСИВНОСТИ РАБОТЫ РОТОРА ГЕЛИКОИДАЛЬНОГО ТИПА С ПОМОЩЬЮ ГИДРОДИНАМИКИ
}

\author{
() К.А. Иванов", Д.А. Иванов, А.П. Руденко, С.Н. Мартыновская \\ Сибирский государственный технологический университет, пр. Мира, 82, \\ Красноярск, 660049 (Россия), e-mail: ivanov.sibstu@yandex.ru
}

В работе рассматривается влияние составляющих абсолютной скорости потока жидкости на процесс перемешивания. Представлены результаты исследования гидродинамики в перемешивающем аппарате с различными перемешивающими устройствами. Показано функциональное преимущество применения ротора геликоидального типа в процессах перемешивания.

Ключевые слова: аппарат, гидродинамика, гомогенность, ИВК, лопастная мешалка, проточная полость, ротор, циркуляция, эпюры скоростей.

\section{Введение}

Исследованию гидродинамики течения жидкости в аппаратах с мешалками посвящено много теоретических и экспериментальных работ [1-13]. Ввиду сложного характера течения жидкости в таких аппаратах авторам указанных работ удалось получить математическое описание распределения скорости только для некоторых сравнительно простых случаев. В большинстве же других случаев приходилось ограничиваться графическим изображением экспериментальных данных. Такие исследования относятся главным образом к вертикальным цилиндрическим аппаратам с мешалками, расположенными на оси аппарата.

Одним из основных параметров, характеризующих интенсивность и эффективность перемешивания, является кратность циркуляции. Под циркуляцией жидкости в аппарате с мешалкой следует понимать перемещение жидкости по замкнутому контуру в соответствии с направлением линий тока [1].

Характер циркуляции жидкости в аппарате с мешалкой зависит главным образом от типа мешалки и от наличия в аппарате вертикальных отражательных планок. Каждая мешалка создает поток жидкости, который, в свою очередь, вызывает циркуляцию во всем объеме аппарата вдоль так называемых циркуляционных петель. Поток жидкости, создаваемый мешалкой, имеет, по меньшей мере, две или чаще всего три составляющих абсолютной скорости. Для оценки работы различных мешалок были введены понятия окружной и радиально-осевой циркуляции [12].

Осевая циркуляция связана с вращением всей массы жидкости вокруг оси вращения мешалки. Радиально-осевая циркуляция связана с насосным действием мешалки и имеет существенное значение для процесса перемешивания, так как при ее отсутствии не может быть и речи о конвективном перемешивании

Иванов Кирилл Александрович - аспирант,

e-mail: www.kir_ill@list.ru

Иванов Дмитрий Александрович - аспирант, e-mail: ivanov.sibstu@yandex.ru

Руденко Анатолий Павлович - заведующий кафедрой технологии конструкционных материалов и машиностроения, доктор технических наук, профессор, тел.: (3912) 12-23-36, e-mail: tolyrudenko@yandex.ru

Мартыновская Светлана Николаевна - доцент, кандидат педагогических наук, e-mail: svetlanamart@yandex.ru в аппарате. Отсюда следует, что на качество перемешивания важное влияние оказывают соотношения окружных и радиально-осевых скоростей. Так, например, при преобладании окружной составляющей абсолютной скорости качество перемешивания снижается из-за уменьшения кратности циркуляции потока жидкости в рабочей полости аппарата. При преобладании радиально-осевой составляющей, наоборот, качество перемешивания улучшается с повышением кратности циркуляции.

\footnotetext{
* Автор, с которым следует вести переписку.
} 


\section{Экспериментальная часть}

С целью исследования составляющих скоростей и результирующей скорости жидкости в аппаратах с мешалками многие авторы $[8,12,14,15]$ использовали различные приборы, начиная с простых трубок скоростного напора до очень сложных изотопных зондов. Для проведения исследований гидродинамики авторами был применен метод зондирования емкостной полости перемешивающего аппарата шаровым зондом, причем полученные результаты были использованы для построения эпюр скоростей. Для проведения исследований была собрана лабораторная установка, включающая в себя перемешивающий аппарат и измерительно-вычислительный комплекс (ИВК). Принципиальный подход к конструированию ИВК предусматривал использование рекомендаций Санкт-Петербургского университета растительных полимеров $[15,16]$.

Для определения эффективности и интенсивности работы ротора геликоидального типа исследования проводились в аппарате с цилиндрическим корпусом, но сначала с применением в качестве перемешивающего устройства - лопастной мешалки. Частота вращения n вала составляла от 100 до 500 об/мин. Анализ гидродинамической картины, создаваемой мешалками, был основан на определении соотношений окружных и осевых скоростей.

\section{Обсуждение результатов}

По полученным данным была произведена математическая обработка, результатом которой явилось нахождение аналитических выражений всех составляющих скоростей течения потока жидкости в проточной полости аппарата.

Для аппарата с цилиндрическим корпусом с лопастной мешалкой уравнения имеют следующий вид:

$$
\begin{gathered}
v_{\text {абс }}=0,351705+13,4799 \cdot R+0,00000238826 \cdot n^{2}- \\
-265,915 \cdot R \cdot H+65,4534 \cdot H^{2} \\
v_{\text {окр }}=0,4061+0,0175187 \cdot n \cdot H+158,712 \cdot R^{2}- \\
-324,705 \cdot R \cdot H+70,1223 \cdot H^{2} \\
v_{\text {рад }}=0,0810246-0,00000923034 \cdot n^{2}+0,0599631 \cdot n \cdot R+ \\
+0,0267665 \cdot n \cdot H-94,9654 \cdot R \cdot H \\
+7,07087 \cdot 10^{-7} \cdot n^{2}-113,295 \cdot R \cdot H+64,1288 \cdot H^{2} \\
v_{\text {осев }}=0,288264+10,6582 \cdot R-7,85223 \cdot H+
\end{gathered}
$$

где $n$ - частота вращения ротора, об/мин; $R$ - радиус рассматриваемого сечения в аппарате, м; $H$ - высота рассматриваемого сечения в аппарате, м.

На основе уравнений (2) и (4) построены с применением программ Excel и «Компас» эпюры окружной и осевой составляющей абсолютной скорости движения жидкости в проточной полости перемешивающего аппарата в четырех рассматриваемых сечениях по высоте аппарата снизу вверх (рис. 1) при частоте вращения вала 100, 300 и 500 об/мин.

Анализируя полученные эпюры скоростей, следует отметить, что наиболее значительное влияние на движение потоков жидкости оказывает окружная составляющая абсолютной скорости. Это влияние прогрессирует с увеличением частоты вращения лопастной мешалки, достигая своего максимального значения в верхней части аппарата (окружная скорость возрастает от $\mathrm{u}_{\text {окр }}=0,4 \mathrm{~m} / \mathrm{c}$ при $\mathrm{n}=100$ об/мин до $\mathrm{u}_{\text {окр }}=1,07 \mathrm{~m} / \mathrm{c}$ при $n=500$ об/мин). Осевая скорость с увеличением частоты вращения ротора возрастает незначительно (от $\mathrm{u}_{\mathrm{oc}}=0,32 \mathrm{м} / \mathrm{c}$ при $\mathrm{n}=100$ об/мин до $\mathrm{u}_{\mathrm{oc}}=0,49 \mathrm{м} / \mathrm{c}$ при $\mathrm{n}=500$ об/мин).

Полученные эпюры скоростей наглядно показывают малую эффективность применения лопастной мешалки для достижения требуемой гомогенности получаемого продукта. 
a)

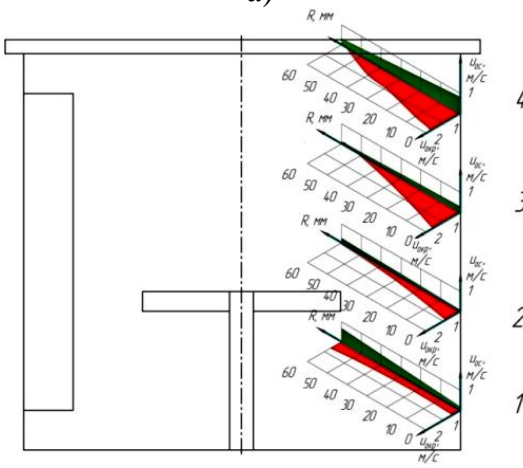

б)

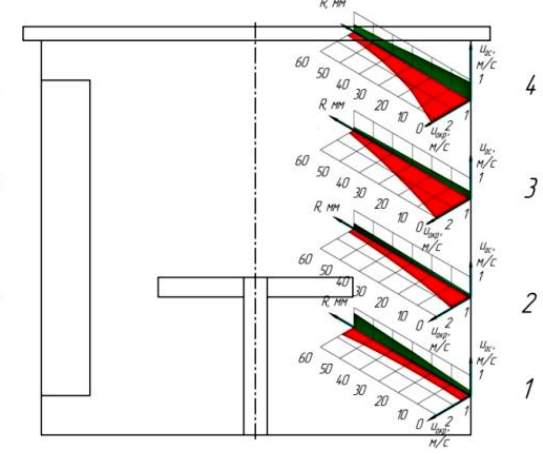

B)

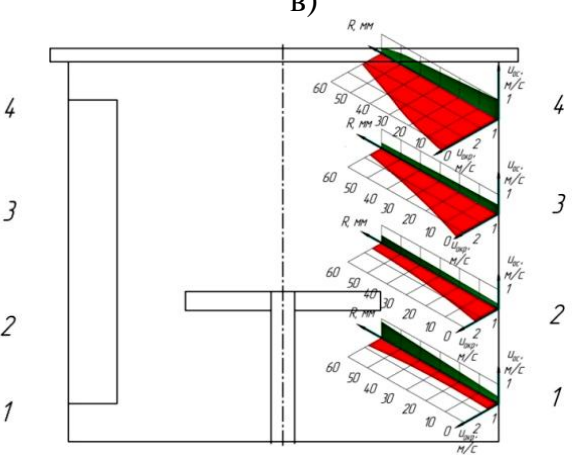

Рис. 1. Схема эпюр скоростей в перемешивающем аппарате с лопастной мешалкой: $\mathrm{a}-\mathrm{n}=100$ об/мин; б 300 об/мин; в - 500 об/мин. 1, 2, 3, 4 - рассматриваемые сечения в аппарате. Зеленое (светлое) поле осевые скорости; красное (темное) поле - окружные скорости

Дальнейшие исследования гидродинамики проводились в аппарате с цилиндрическим корпусом, но в качестве перемешивающего органа использовался ротор геликоидального типа.

В этом случае в ходе обработки экспериментальных данных также были получены аналитические зависимости (5-8) и построены эпюры окружной и осевой составляющей абсолютной скорости движения жидкости в проточной полости цилиндрического аппарата в четырех рассматриваемых сечениях при частоте вращения ротора 100, 300 и 500 об/мин (рис. 2).

$$
\begin{gathered}
v_{\text {абсс }}=-0,359+31,027 \cdot R+16,703 \cdot H+3,102 \cdot 10^{-6} \cdot n^{2}- \\
-0,018 \cdot n \cdot H-312,482 \cdot R^{2}-59,26 \cdot H^{2} \\
v_{\text {окр }}=-1,114+2,086 \cdot 10^{-3} \cdot n+34,912 \cdot R+11,949 \cdot H- \\
-33,952 \cdot 10^{-3} \cdot n \cdot H-412,727 \cdot R^{2}+215,562 \cdot R \cdot H-78,371 \cdot H^{2} \\
v_{\text {рад }}=0,199-1,716 \cdot 10^{-3} \cdot n+3,351 \cdot R+14,413 \cdot H+ \\
+4,156 \cdot 10^{-7} \cdot n^{2}+2,687 \cdot 10^{-3} \cdot n \cdot R+0,0166 \cdot n \cdot H- \\
-58,438 \cdot n^{2}+22,266 \cdot R \cdot H-94,696 \cdot H^{2} \\
v_{\text {oсев }}=0,559+1,683 \cdot 10^{-3} \cdot n^{2}-44,073 \cdot R \cdot H
\end{gathered}
$$

где $n$ - частота вращения ротора, об/мин; $R$ - радиус рассматриваемого сечения в аппарате, м; $H$ - высота рассматриваемого сечения в аппарате, м.

При анализе эпюр установлено, что значение окружной скорости с повышением частоты вращения ротора увеличивается, достигая максимального значения $\mathrm{u}_{\text {окр }}=0,59 \mathrm{~m} / \mathrm{c}$ при $\mathrm{n}=500$ об/мин. Кроме того, наблюдается изменение направления движения потока жидкости на противоположное под действием отражательных планок, установленных на стенке корпуса цилиндрического аппарата. Рассматривая величины осевых скоростей, можно сделать вывод о том, что с увеличением частоты вращения ротора происходит рост осевой скорости от 0,39 м/с при $\mathrm{n}=100$ об/мин до 0,79 м/с при $\mathrm{n}=500$ об/мин. При этом максимальное значение скорости достигается у стенки аппарата, где располагаются вертикальные отражательные планки. В целом применение ротора геликоидального типа позволяет значения окружной скорости снизить до $80 \%$, а значения осевой скорости - повысить до $40 \%$.

Проводя сравнение вышеописанных перемешивающих органов, можно сделать заключение о том, что наиболее предпочтительным для процессов перемешивания является использование ротора геликоидального типа, так как он создает в рабочей полости аппарата необходимую гидродинамическую картину движения потоков жидкости, что является одним из важнейших условий при получении продукции требуемого качества. 
a)

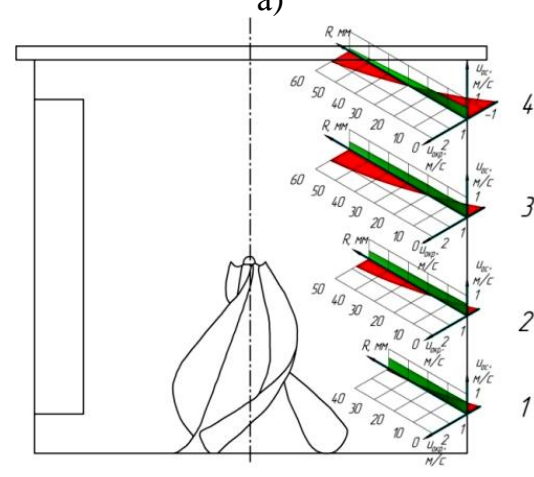

б)

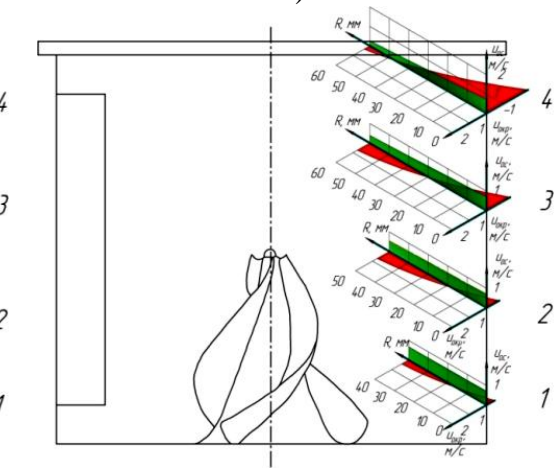

B)

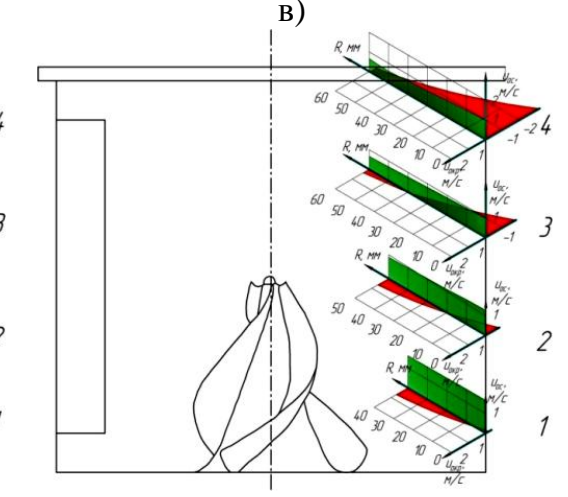

Рис. 2. Схема эпюр скоростей в аппарате стандартной конструкции с ротором геликоидального типа: $\mathrm{a}-\mathrm{n}=100$ об/мин; б - 300 об/мин; в - 500 об/мин. 1, 2, 3, 4 - рассматриваемые сечения в аппарате. Зеленое (светлое) поле - осевые скорости; красное (темное) поле - окружные скорости

\section{Bbыводы}

На основании проведенных исследований и полученных результатов можно сделать вывод о том, что в аппарате с цилиндрическим корпусом при использовании в качестве перемешивающего органа ротора геликоидального типа наблюдается преобладание радиально-осевых течений жидкости по сравнению с окружными течениями. Возникновение и стабильное существование данной гидродинамической картины в проточной полости аппарата способствует повышению интенсивности циркуляции и, как закономерный результат, способствует увеличению качественных показателей готового продукта.

\section{Список литературы}

1. Стренк Ф. Перемешивание и аппараты с мешалками. Л., 1975. 384 с.

2. Брагинский Л.Н., Бегачев В.И., Барабаш В.М. Перемешивание в жидких средах. Л., 1984. 336 с.

3. Васильцов 3.А., Ушаков В.Г. Аппараты для перемешивания жидких сред. Л., 1989. 271 с.

4. Штербачек 3., Тауск П. Перемешивание в химической промышленности. Л., 1963. 416 с.

5. Холланд Ф., Чапман Ф. Химические реакторы и смесители для жидкофазных процессов. М., 1974. 208 с.

6. Гзовский С.Я. Основные элементы перемешивающих аппаратов // Химическое машиностроение. 1959. №6. С. 13-14.

7. Гзовский С.Я. Конструктивное оформление процесса перемешивания // Химическое машиностроение. 1960. №1. C. 17-19.

8. Nagata S., Janagimoto M., Jokojama T. A studie on the mixing of highviscous liquid // Kagaky Kogaky. 1957. Vol. 21, N5. Pp. 278-286.

9. Aiba S. Flow pattems of liquids in agitated vessels // AIChE Journal. 1958. Vol. 4, N4. Pp. 485-491.

10. Глухов В.П., Брагинский Л.Н., Павлушенко И.С., Павлов Н.Г., Бегачев В.И. Распределение скоростей в аппаратах со скребковыми мешалками // Теория и практика перемешивания в жидких средах. М., 1973. С. 78-80.

11. Костин Н.М., Павлушенко И.С. Изучение процесса перемешивания. Определение скорости движения жидкости в аппаратах с пропеллерной мешалкой // Сборник трудов ЛТИ. 1957. №31. С. 131-144.

12. Nagata S., Yokoyama K., Ujihara M. Flow patterns of liquid in a cylindrical mixing vessel without bafflers // Chemical engineering. 1959. Vol. 23, N3. Pp. 130-137.

13. Barnea E., Mizrahi J. On the «effective» viscosity of liquid - liquid dispersions // Ind. and Eng. Chem. Fundam., 1976. Vol. 15, N2. Pp. 120-125.

14. Гзовский С.Я. Основные элементы перемешивающих аппаратов // Химическое машиностроение. 1959 . №6. C. $13-14$.

15. Терентьев О.А. Гидродинамика волокнистых суспензий. Л., 1972. 142 с.

16. Александров А.В. Основы теории структурообразования бумажной массы в процессе напуска на сеточную часть бумагоделательной машины : дисс. ... докт. техн. наук. Л., 1988. 398 с. 
Ivanov K.A., Ivanov D.A., Rudenko A.P., Martynovskaya S.N. DETERMINATION OF EFFICIENCY AND INTENSITY OF WORK OF A ROTOR OF THE HELICOID TYPE BY MEANS OF HYDRODYNAMICS

Siberian State Technological University, pr. Mira, 82, Krasnoyarsk, 660049 (Russia), e-mail: ivanov.sibstu@yandex.ru

In work influence of components of absolute speed of a stream of liquid on mixing process is considered. Results of research of hydrodynamics in the mixing device with various mixing devices are presented. Functional advantage of application of the Helicoid rotor type in the process of mixing is shown. diagram.

Keywords: cavity, circulation, device, flowing cavity, homogeneity, hydrodynamics, MCC, paddle stirrer, rotor, speeds

\section{References}

1. Strenk F. Peremeshivanie i apparaty s meshalkami. [Stirring machines with agitators.]. Leningrad, 1975, 384 p. (in Russ.).

2. Braginskii L.N., Begachev V.I., Barabash V.M. Peremeshivanie v zhidkikh sredakh. [Stirring in liquid media.]. Leningrad, 1984, 336 p. (in Russ.).

3. Vasil'tsov Z.A., Ushakov V.G. Apparaty dlia peremeshivaniia zhidkikh sred. [Apparatus for mixing fluids.]. Leningrad, 1989, 271 p. (in Russ.).

4. Shterbachek Z., Tausk P. Peremeshivanie $v$ khimicheskoi promyshlennosti. [Stirring in chemical industry.]. Leningrad, 1963, 416 p. (in Russ.).

5. Kholland F., Chapman F. Khimicheskie reaktory i smesiteli dlia zhidkofaznykh protsessov. [Chemical reactors and mixers for liquid-phase processes]. Moscow, 1974, 208 p. (in Russ.).

6. Gzovskii S.Ia. Khimicheskoe mashinostroenie, 1959, no. 6, pp. 13-14. (in Russ.).

7. Gzovskii S.Ia. Khimicheskoe mashinostroenie, 1960, no. 1, pp. 17-19. (in Russ.).

8. Nagata S., Janagimoto M., Jokojama T. Kagaky Kogaky, 1957, vol. 21, no. 5, pp. 278-286.

9. Aiba S. AIChE Journal, 1958, vol. 4, no. 4, pp. 485-491.

10. Glukhov V.P., Braginskii L.N., Pavlushenko I.S., Pavlov N.G., Begachev V.I. Teoriia i praktika peremeshivaniia v zhidkikh sredakh. [Theory and Practice of mixing fluids.]. Moscow, 1973, pp. 78-80. (in Russ.).

11. Kostin N.M., Pavlushenko I.S. Cbornik trudov LTI, 1957, no. 31, pp. 131-144. (in Russ.).

12. Nagata S., Yokoyama K., Ujihara M. Chemical engineering, 1959, vol. 23, no. 3, pp. 130-137.

13. Barnea E., Mizrahi J. Ind. and Eng. Chem. Fundam., 1976, vol. 15, no. 2, pp. 120-125.

14. Gzovskii S.Ia. Khimicheskoe mashinostroenie, 1959, no. 6, pp. 13-14. (in Russ.).

15. Terent'ev O.A. Gidrodinamika voloknistykh suspenzii. [Hydrodynamics of fiber suspensions.]. Leningrad, 1972, 142 p. (in Russ.).

16. Aleksandrov A.V. Osnovy teorii strukturoobrazovaniia bumazhnoi massy v protsesse napuska na setochnuiu chast' bumagodelatel'noi mashiny : diss. .... dokt. tekhn. nauk. [Fundamentals of the theory of structure formation in the pulp lapping on the screen portion godelatelnoy boom-machine: the dissertation of the doctor of technical sciences.]. Leningrad, 1988, 398 p. (in Russ.). 
